\title{
Editoriali
}

\section{Interazione tra fattori biologici e sociali in psichiatria: il modello della «riserva cerebrale»}

\author{
MARIO MAJ
}

L'interazione tra fattori biologici e sociali in psichiatria è stata oggetto di numerosi contributi nel corso degli ultimi vent'anni. Teorie che postulano un ruolo scatenante degli eventi stressanti in soggetti portatori di una vulnerabilità biologica specifica, o che ipotizzano l'intervento di fattori socio-ambientali nel condizionare in senso patologico lo sviluppo funzionale del sistema nervoso centrale, sono state formulate soprattutto per quanto riguarda la schizofrenia, ma anche a proposito delle sindromi affettive maggiori.

Nella letteratura in lingua italiana esistono diversi articoli in cui vengono esposte ed esaminate criticamente queste teorie (Maj et al., 1985; Faravelli e Pallanti, 1990; Balestrieri, 1992). Piuttosto che discuterle ancora una volta, quindi, preferisco presentare in questa nota un modello completamente nuovo per il lettore italiano, detto della «riserva cerebrale», il quale ipotizza un'interazione tra fattori biologici e sociali nel determinare la precocità dell'insorgenza del deficit cognitivo conseguente ad un danno cerebrale acquisito.

È noto da tempo che i circuiti cerebrali implicati nelle funzioni cognitive presentano una certa ridondanza, la quale permette l'attivazione di circuiti alternativi in caso di lesioni di varia natura, prevenendo o ritardando l'insorgenza di un deficit cognitivo evidente sul piano clinico o rilevabile mediante tests neuropsicologici.

L'esistenza di questa capacità compensatoria da parte del cervello è stata dimostrata con ricerche condotte mediante la tomografia ad emissione di positroni e lo studio del flusso ematico cerebrale (Grady et al., 1988), da cui è risultato che nella malattia di

Indirizzo per la corrispondenza: Prof. M. Maj, Istituto di Psichiatria, Prima Facoltà Medica, Università di Napoli, Largo Madonna delle Grazie, 80138 Napoli.

Fax $(+39)$ 081-449.938.
Alzheimer la comparsa di un danno metabolico neocorticale precede di molti mesi o anche di anni l'insorgenza di una compromissione clinicamente significativa delle funzioni cognitive.

L'ipotesi a cui facciamo qui riferimento postula che la suddetta ridondanza dei circuiti cerebrali (la «riserva cerebrale») sia di entità differente da un individuo all'altro, e possa essere condizionata sia da fattori organici (ad esempio, traumi cranici che riducono l'estensione del tessuto cerebrale funzionante) che da fattori psicosociali (ad esempio, una carente stimolazione e un ridotto esercizio intellettuale, che limitano l'estensione e/o l'efficienza delle connessioni sinaptiche neuronali a livello cerebrale).

L'evidenza che ci ha indotto a prendere in esame quest'ipotesi è derivata dallo studio cross-culturale sugli aspetti neuropsichiatrici dell'infezione da HIV-1 recentemente promosso dall'Organizzazione Mondiale della Sanità (Maj et al., 1991). Dalla fase trasversale di tale studio, infatti, è emerso che in alcuni contesti geografici la compromissione della prestazione ad una batteria di tests neuropsicologici (cioè, un punteggio almeno due deviazioni standard al di sotto della media dei controlli in almeno tre su dieci tests) è significativamente più frequente nei sieropositivi asintomatici con basso livello di istruzione (al di sotto del $25^{\circ}$ percentile della media dei controlli in quel dato centro) rispetto ai controlli sieronegativi con lo stesso livello di istruzione, mentre non c'è differenza significativa tra sieropositivi asintomatici e sieronegativi con livello di istruzione elevato (Maj et al., in stampa).

Per spiegare questo dato, noi abbiamo ipotizzato che un basso livello di istruzione rappresenti un "marcatore» di una ridotta riserva cerebrale, associandosi quindi ad una comparsa più precoce del deficit cognitivo conseguente al danno cerebrale prodotto dall'infezione da HIV-1. 
Esattamente, un basso livello di istruzione potrebbe essere: 1) una causa di ridotta ridondanza dei circuiti cerebrali (in quanto comporta una carente stimolazione e un ridotto esercizio intellettuale); 2) un effetto della ridotta ridondanza (in quanto i soggetti che già presentano una riserva cerebrale diminuita potrebbero avere una maggiore difficoltà ad andare avanti negli studi); 3) un correlato di una situazione ambientale in cui è elevata l'incidenza di altri fattori (traumi ostetrici, abuso di sostanze, malnutrizione) verosimilmente in grado di ridurre la ridondanza dei circuiti cerebrali.

La riflessione sul dato suddetto ci ha spinto a cercare nella letteratura scientifica altre evidenze di una relazione tra livello di istruzione ed età di insorgenza di condizioni morbose caratterizzate da un deficit cognitivo. In questa sede facciamo brevemente riferimento a due lavori recenti, entrambi riguardanti la malattia di Alzheimer.

Il primo lavoro è una meta-analisi della letteratura concernente il ruolo dei traumi cranici come fattore di rischio per la comparsa della malattia di Alzheimer (Mortimer et al., 1991). È noto che tale ruolo è documentato da varie evidenze (Roberts et al., 1990), e che esistono anche studi secondo cui l'esordio della malattia di Alzheimer è più precoce nei soggetti che hanno subito in passato un grave trauma cranico (Gedye et al., 1989), la qual cosa giustifica l'inclusione dei traumi in questione tra $i$ fattori capaci di ridurre la riserva cerebrale. Ciò che più ci interessa nell'ambito della nostra discussione, però, è il fatto che il periodo di latenza compreso tra il trauma cranico e l'esordio della malattia di Alzheimer risulta essere, dalla suddetta meta-analisi della letteratura, significativamente ridotto nei soggetti con basso livello di istruzione (osservazione compatibile con l'idea che un livello di istruzione modesto rappresenti un indicatore di una riserva cerebrale ridotta).

Il secondo lavoro che voglio citare riporta i risultati di uno studio del flusso ematico cerebrale nelle regioni parieto-temporali di tre gruppi di soggetti con malattia di Alzheimer, paragonabili per durata della malattia ed entità del deficit cognitivo, ma differenti per livello di istruzione (Stern et al., 1991). Questo studio ha messo in evidenza un più grave difetto di perfusione nei soggetti con livello di istruzione più elevato (osservazione spiegabile ammettendo che un alto livello di istruzione rappresenti un indicatore di un'aumentata riserva cerebrale, per effetto della quale ad un maggior danno metabolico non corrisponde un più accentuato deficit cognitivo).

Naturalmente, tutte le evidenze suddette richiedo- no ulteriori conferme. Io ritengo, però, che la probabile relazione tra livello di istruzione e riserva cerebrale vada segnalata all'attenzione degli studiosi, per le sue affascinanti implicazioni sul piano teorico. Infatti, ammettere che il basso livello di istruzione rappresenti una possibile causa di limitazione della riserva cerebrale significa riconoscere che fattori psicosociali possano agire ad un medesimo livello di quelli organici (qui non si parla di «parallelismo» mentecervello!) su una situazione anatomo-funzionale cerebrale.

\section{BIBLIOGRAFIA}

Balestrieri A. (1992). Darwinismo neuronale, epigenesi selettiva, personalità e schizofrenia. Rivista di Psichiatria 27, 1-5.

Faravelli C. \& Pallanti S. (1990). Accadimenti ambientali e malattia. In Psichiatria Medica (ed. G. B. Cassano), pp. 157-170. UTET: Torino.

Gedye A., Beattie B. L., Tuokko H., Horton A. \& Korsarek E. (1989). Severe head injury hastens age of onset of Alzheimer's disease. Journal of the American Geriatrics Society 37, 970-973.

Grady C. L., Haxby J. V., Horwitz B., Sundaram M., Berg G., Schapiro M., Friedland R. P. \& Rapoport S. I. (1988). Longitudinal study of the early neuropsychological and cerebral metabolic changes in dementia of the Alzheimer type. Journal of Clinical and Experimental Neuropsychology 10, 576-596.

Maj M., Gritti P. \& Galderisi S. (1985). La «diathesis-stress theory» della schizofrenia: basi teoriche ed implicazioni terapeutiche. Neurologia, Psichiatria, Scienze Umane 5, 140-156.

Maj M., Janssen R., Satz P., Zaudig M., Starace F., Boor D., Sughondhabirom B., Bing E. G., Luabeya M., Ndetei D., Riedel R., Schulte G. \& Sartorius N. (1991). The World Health Organization's cross-cultural study on neuropsychiatric aspects of infection with the human immunodeficiency virus 1 (HIV-1). Preparation and pilot phase. British Journal of Psychiatry 159, 351-356.

Maj M., Satz P., Janssen R., Zaudig M., Starace F., d'Elia L., Sughondhabirom B., Mussa M., Bing E. G., Naber D., Ndetei D., Schulte G. \& Sartorius N. (in stampa). WHO Neuropsychiatric AIDS Study, cross-sectional phase. II. Neuropsychological and neurological findings. Archives of General Psychiatry.

Mortimer J. A., van Duijn C. M., Chandra V., Fratiglioni L., Graves A. B., Heyman A., Jorm A. F., Kokmen E., Rocca W. A., Shalat S. L., Soininen H. \& Hofman A. (1991). Head trauma as a risk factor for Alzheimer's disease: a collaborative re-analysis of case-control studies. International Journal of Epidemiology 20, S28-S35.

Roberts G. W., Allsop D. \& Bruton C. (1990). The occult aftermath of boxing. Journal of Neurology, Neurosurgery and Psychiatry 53, 373-378.

Stern Y., Alexander G., Prohovnik I. \& Mayeux R. (1991). Education provides a cognitive reserve against the clinical manifestations of Alzheimer's disease: evidence form regional cerebral blood flow. Society for Neuroscience Abstracts 17, 1258. 